\title{
Guest Editorial Nonlinear Optimization of Communication Systems
}

$\mathbf{L}$ INEAR programming and other classical optimization techniques have found important applications in communication systems for many decades. Recently, there has been a surge in research activities that utilize the latest developments in nonlinear optimization to tackle a much wider scope of work in the analysis and design of communication systems. These activities involve every "layer" of the protocol stack and the principles of layered network architecture itself, and have made intellectual and practical impacts significantly beyond the established frameworks of optimization of communication systems in the early 1990s. These recent results are driven by new demands in the areas of communications and networking, as well as new tools emerging from optimization theory. Such tools include the powerful theories and highly efficient computational algorithms for nonlinear convex optimization, together with global solution methods and relaxation techniques for nonconvex optimization.

The phrase "nonlinear optimization of communication systems" carries at least three different meanings. In the most straightforward way, an analysis or design problem in a communication system may be formulated as minimizing a cost function, or maximizing a utility function, or determining feasibility over a set of variables confined within a constraint set. In a more subtle and recent approach, a given network protocol may be reverse-engineered as a distributed algorithm solving an implicit, global optimization problem. In yet another approach, the underlying theory of a network control method or a communication strategy may be generalized using nonlinear optimization techniques, thus extending the scope of applicability of the theory.

Over the last few years, the nonlinear optimization framework has been successfully applied to a wide range of problems in communication systems, from the high-speed Internet core to wireless ad hoc networks, from coding and equalization to broadband access, and from information theory to network topology models. This Special Issue further showcases the roles of nonlinear optimization in our understanding and control of communication systems: more than a powerful modeling and design tool, it further provides a rigorous mentality and a common language for researchers to think about both performance and architectural issues.

Digital Object Identifier 10.1109/JSAC.2006.879335
We have received a large number of submissions, 95 papers by the deadline of September 2005. After extensive and careful reviews followed by editorial board meetings, and under the constraint of total page count in a J-SAC Special Issue, we accepted 17 papers that are of both the highest quality and the best fit with the theme of this Special Issue. Guest Editors have also written three tutorials on the mathematical techniques and general themes that will help the readers further appreciate the wealth of materials covered in this Special Issue. These 20 papers provide an exciting view on the wide and expanding intellectual landscape of the subject. They cover a variety of topics on the applications of nonlinear optimization and Lagrange duality to many aspects of communication systems analysis and design, including parallel and distributed optimization techniques or problems whose data or control mechanisms are spatially distributed.

This Special Issue opens with three tutorial papers. In "An Introduction to Convex Optimization for Communications and Signal Processing," Luo and Yu first survey the basics of convex optimization, especially second-order-cone and semidefinite programming, then demonstrate several applications of conic programming and robust optimization to multiuser communications problems. In "A Tutorial on Decomposition Methods for Network Utility Maximization," Palomar and Chiang first survey the basics of decomposition theory and the construction of a variety of distributed algorithms based on the elements of primal and dual decomposition, then illustrate the search for alternative decompositions and hidden decomposability structures. In "A Tutorial on Cross-Layer Optimization in Wireless Networks," Lin et al. first survey the recent developments of using optimization theory for wireless resource allocation and "loosely coupled" cross-layer design, then discuss the open issues involving distributed and imperfect scheduling in medium access control (MAC) design.

The 17 contributed papers are grouped into four clusters according to topics. First are four papers on Optimization of Wired Networks. In "Layered Multicast Rate Control Based on Lagrangian Relaxation and Dynamic Programming," Kar and Tassiulas formulate a rate control problem for a layered multicast problem as a utility maximization that involves integer constraints. This paper applies dual decomposition to the Lagrangian relaxation of the problem and proves, using dynamic programming techniques, that the dual algorithm is approximately optimal. In "Distributed Utility Maximization 
for Network Coding Based Multicasting: A Shortest Path Approach," Wu and Kung develop a utility maximization model for multicast sessions using network coding. The structure of the single-source multidestination multicast network is exploited using a dual decomposition method and a shortest path algorithm. In "Super-Fast Delay Tradeoffs for Utility Optimal Fair Scheduling in Wireless Networks," Neely considers the problem of utility-delay tradeoff in scheduling with time-varying channels, showing that a simple set of algorithms can achieve the best tradeoff of utility coming within $\mathcal{O}(1 / V)$ of optimum and delay growing logarithmically with parameter $V$. In "Distributed Nonlinear Integer Optimization for Data-Optical Internetworking," Elwalid et al. address the practical problem of bandwidth provisioning in networks, where the core of the network operate at substantially higher speeds than the periphery. The authors develop a bandwidth provisioning scheme based on Bender's decomposition technique whose performance is explicitly analyzed and solution implemented in a tractable, distributed manner.

Following the above are four papers on Optimization of Wireless Networks. In "Joint Congestion Control, Routing, and MAC for Stability and Fairness in Wireless Networks," Eryilmaz and Srikant propose a joint scheduling, routing, and congestion control mechanism that asymptotically guarantees stability of buffers and optimality of a primal-dual congestion controller. Stability is proved through a Lyapunov function and La Salle's invariance principle. In "Adaptive Sensor Activity Control in Many-to-One Sensor Networks," Hu et al. consider the problem of adaptively activating sensors in a way that maximizes sensor net lifetime subject to a constraint on sensing quality. This paper proposes algorithms and proves their optimality under various conditions on the knowledge of the total number of sensors and the number of currently active sensors. In "Mathematical Decomposition Techniques for Distributed Cross-Layer Optimization of Data Networks," Johansson et al. apply the techniques of primal and dual decompositions to distributively solve two resource allocation problems, one in wireless networks with orthogonal channels and network-wide resource constraints, and another in wireless networks with spatial-reuse time-division multiple-access (TDMA) physical layer. In "Cross-Layer Rate Optimization for Proportional Fairness in Multihop Wireless Networks With Random Access," Wang and Kar develop a rate control algorithm for multihop wireless networks such that both the end-to-end data rate of users and the static probability of link activation are controlled to maximize system utility. The authors exploit the special structure of the optimization problem to map it to an equivalent convex problem.

Then, we have four papers on Optimization in Communication Theory and Signal Processing. In "Robust Linear Receivers for Multiaccess Space-Time Block-Coded MIMO Systems: A Probabilistically Constrained Approach," Rong et al. consider the problem of designing multiuser multiple-input-mul- tiple-output (MIMO) receivers, which are robust against imperfect channel state estimation. The authors model robustness using probabilistic chance constraints and reformulate the problem as a convex one under Gaussian assumption. In "Multiuser Margin Optimization in Digital Subscriber Line (DSL) Channels," Panigrahi et al. tackle the physical-layer power-spectrum-density optimization problem for the digital subscriber line (DSL) application, where crosstalk interference between neighboring lines is a major problem. The authors formulate a novel margin maximization problem and provide optimization solutions from this perspective. In "Optimizing Power Efficiency of OFDM Using Quantized Channel State Information," Marqués et al. develop loading algorithms to solve the problem of minimizing transmit power under rate and error probability constraints. Deterministic, statistical, and quantized channel state information are considered. In "Queue Proportional Scheduling via Geometric Programming in Fading Broadcast Channels," Seong et al. use the convexity of the broadcast channel capacity region and geometric programming in convex form to illustrate that several traffic scheduling problems can be formulated as convex optimization. The authors also prove that the resulting scheduling is throughput optimal.

The last set of five papers deals with Optimization in Information, Coding, and Queueing Theories. In "Nonlinear Programming Approaches to Decoding Low-Density ParityCheck Codes," Yang et al. develop nonlinear optimization-based decoding algorithms for low-density parity-check codes. The authors explore the use of the branch-and-bound method in decoding and introduce a quadratic programming technique to improve the efficiency of the decoding algorithm. In "Optimal Bandwidth Allocation in a Delay Channel," Ehsan and Liu consider the problem of allocating bandwidth among multiple queues so as to minimize the total packet holding cost. The results are derived for the case with two queues. The main contribution of this paper is to show that if the holding cost is of certain type (nondecreasing, supermodular, and superconvex), then the optimal policy for allocating over $M$ slots reduces to sequentially allocating one slot optimally at a time. In "Optimized Transmission for Fading Multiple Access and Broadcast Channels With Multiple Antennas," Mohseni et al. consider the problem of optimal resource allocation from an information-theoretic point of view. The authors use convex optimization to characterize the capacity and power regions for the fading multiple-access channel and fading broadcast channel with additive Gaussian noise and multiple transmit and receive antennas. In "An Iterative Water-Filling Algorithm for Maximum Weighted Sum-Rate of Gaussian MIMO-BC," Kobayashi and Caire provide an efficient numerical algorithm to solve for the optimal input covariance for the Gaussian multiple-access channel and the Gaussian broadcast channel with multiple antennas. The authors use an iterative water-filling algorithm to find the entire capacity region for both cases. In 
"Error Exponents for Channel Coding With Application to Signal Constellation Design," Huang et al. show that, under general assumptions on the channel statistics, the input distributions that maximize the random coding exponent have a finite number of mass points. The authors also introduce a new class of cutting-plane algorithms to efficiently compute the optimal distributions.

We are excited to put together, within one year since the submission deadline, a J-SAC Special Issue whose high quality is evidenced by these papers between the covers. This would have been impossible without all those who contributed their research results, hundreds of diligent reviewers from the research community, and the J-SAC Editorial Board and Senior Editor, M. Steenstrup. We hope you will enjoy this Special Issue devoted to the exciting field of Nonlinear Optimization of Communication Systems as much as we do.

\author{
Mung Chiang, Lead Guest Editor \\ Princeton University \\ Department of Electrical Engineering and \\ Program in Applied and Computational \\ Mathematics \\ Princeton, NJ 08544 USA \\ STEVEN H. Low, Guest Editor \\ California Institute of Technology \\ Department of Computer Science and \\ Department of Electrical Engineering \\ Pasadena, CA 91125 USA
ZHI-QUAN LUO, Guest Editor
University of Minnesota
Department of Electrical and Computer Engineering
Minneapolis, MN 55455 USA \\ NESS B. ShrofF, Guest Editor \\ Purdue University \\ School of Electrical and Computer Engineering \\ West Lafayette, IN 47907 USA \\ WEI YU, Guest Editor \\ University of Toronto \\ The Edward S. Rogers Sr. Department of Electrical \\ and Computer Engineering \\ Toronto, ON M4S 3G5, Canada \\ M. SteEnStRuP, J-SAC Board Representative
}

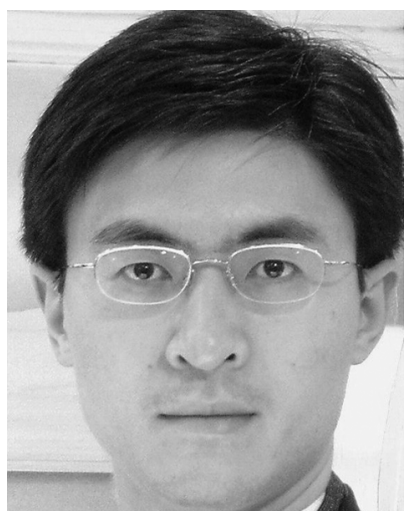

Mung Chiang (S'00-M'03) received the B.S. (Hon.) degree in electrical engineering and mathematics, and the M.S. and Ph.D. degrees in electrical engineering from Stanford University, Stanford, CA, in 1999, 2000, and 2003, respectively.

$\mathrm{He}$ is an Assistant Professor of Electrical Engineering, and an affiliated faculty of Applied and Computational Mathematics at Princeton University. He conducts research in the areas of optimization of communication systems, analytic foundations of network architectures, algorithms in broadband access, and information theory.

Prof. Chiang has been awarded a Hertz Foundation Fellow and received the Stanford University School of Engineering Terman Award, the SBC Communications New Technology Introduction Contribution Award, the National Science Foundation CAREER Award, and the Princeton University Howard B. Wentz Junior Faculty Award. He is the Lead Guest Editor of the IEEE JOURNAL OF SElECTED AREAS IN COMMUNICATIONS (Special Issue on Nonlinear Optimization of Communication Systems), a Guest Editor of the IEEE TRANSACTIONS ON INFORMATION THEORY and IEEE/ACM TRANSACTIONS ON NETWORKING (Joint Special Issue on Networking and Information Theory), and the Program Co-Chair of the 38th Conference on Information Sciences and Systems. 


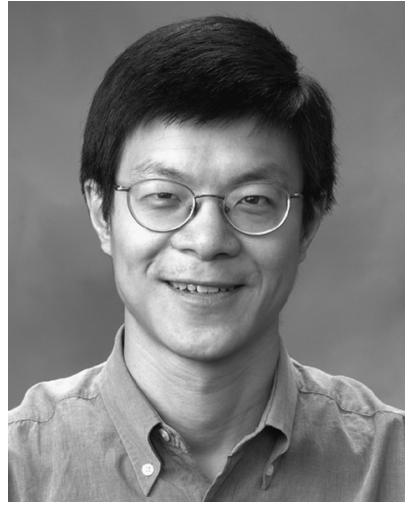

Steven H. Low (M'92-SM'99) received the B.S. degree from Cornell University, Ithaca, NY, and the M.S. and Ph.D. degrees from the University of California at Berkeley, all in electrical engineering.

He is an Associate Professor at the California Institute of Technology (Caltech), Pasadena, and a Senior Fellow of the University of Melbourne, Melbourne, Australia. He was with AT\&T Bell Laboratories, Murray Hill, NJ, from 1992 to 1996, and with the University of Melbourne from 1996 to 2000.

Dr. Low was a corecipient of the IEEE William R. Bennett Prize Paper Award in 1997 and the 1996 R\&D 100 Award. He is on the Editorial Boards of the IEEE/ACM TRANSACTIONS ON NETWORKING, ACM Computing Surveys, NOW Foundations and Trends in Networking, and is a Senior Editor of the IEEE JOURNAL ON SELECTED AREAS In COMMUNICATIONS.

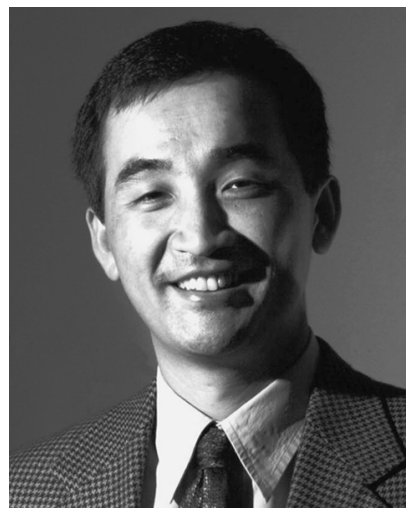

Zhi-Quan (Tom) Luo (SM'03) received the B.Sc. degree in mathematics from Peking University, Peking, China, in 1984. From 1985 to 1989, he studied at the Operations Research Center and the Department of Electrical Engineering and Computer Science, Massachusetts Institute of Technology, Cambridge, where he received the Ph.D. degree in operations research.

During the academic year of 1984 to 1985, he was with the Nankai Institute of Mathematics, Tianjin, China. In 1989, he joined the Department of Electrical and Computer Engineering, McMaster University, Hamilton, ON, Canada, where he became a Professor in 1998 and held the Canada Research Chair in Information Processing since 2001. Since April 2003, he has been a Professor with the Department of Electrical and Computer Engineering and holds an endowed ADC Research Chair in Wireless Telecommunications with the Digital Technology Center, University of Minnesota. His research interests lie in the union of large-scale optimization, information theory and coding, data communications, and signal processing.

Prof. Luo received an IEEE Signal Processing Society Best Paper Award in 2004. He is a member of the Society for Industrial and Applied Mathematics (SIAM) and MPS. He is also a member of the Signal Processing for Communications (SPCOM) and Signal Processing Theory and Methods (SPTM) Technical Committees of the IEEE Signal Processing Society. From 2000 to 2004, he served as an Associate Editor for the IEEE TRANSACTIONS ON SignAL PROCESSING and Mathematics of Computation. He is presently serving as an Associate Editor for several international journals including SIAM Journal on Optimization and Mathematics of Operations Research.

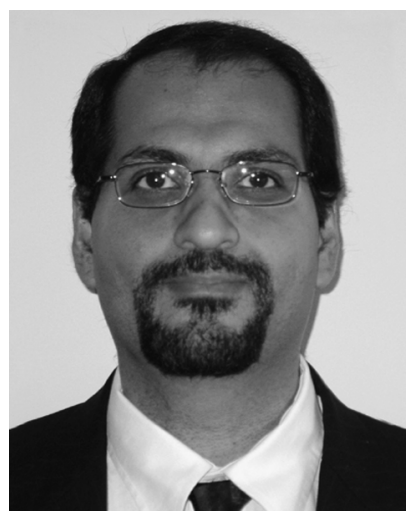

Ness B. Shroff (S'91-M'93-SM'01) received the Ph.D. degree from Columbia University, New York, in 1994.

He joined Purdue University, West Lafayette, IN, in 1994, where he is currently Professor of Electrical and Computer Engineering and director of a university-wide center on wireless systems and applications. His research is funded by various companies such as Motorola, Intel, Hewlett Packard, Nortel, AT\&T, BAE systems, and L. G. Electronics; and government agencies such as the National Science Foundation (NSF), Defense Advanced Research Projects Agency (DARPA), Indiana Department of Transportation, and the Indiana 21st Century Fund. His research interests span the areas of wireless and wireline communication networks. He is especially interested in fundamental problems in the design, performance, control, and security of these networks.

Dr. Shroff received the IEEE INFOCOM 2006 Best Paper Award, the 2005 Best Paper of the Year Award for the Journal of Communications and Networking, the 2003 Best Paper of the Year Award for Computer Networks, and the NSF CAREER award in 1996 (his IEEE INFOCOM 2005 paper was also selected as one of two runner-up papers for the best paper award). He is an Editor for IEEE/ACM TRANSACTIONS ON NETWORKING and the Computer Networks Journal, and past Editor of the IEEE COMMUNICATIONS LETTERS. He has served on the technical and executive committees of several major conferences and workshops. He was the Technical Program Co-Chair of IEEE INFOCOM'03, the premier conference in communication networking. He was also the Conference Chair of the 14th Annual IEEE Computer Communications Workshop (CCW'99), the Program Co-Chair for the Symposium on High-Speed Networks, GLOBECOM 2001, and the Panel Co-Chair for ACM MobiCom'02. He was also a co-organizer of the NSF Workshop on Fundamental Research in Networking, Arlie House, VA, in 2003. 


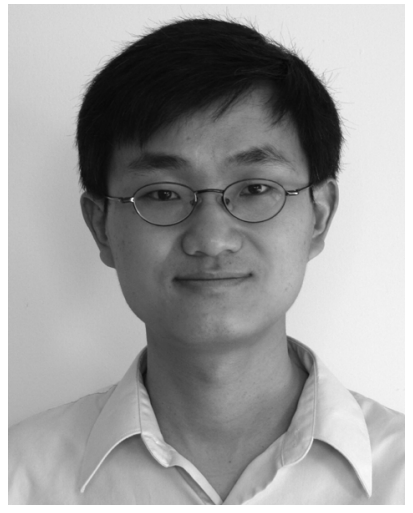

Wei Yu (S'97-M'02) received the B.A.Sc. degree in computer engineering and mathematics from the University of Waterloo, Waterloo, ON, Canada, in 1997, and the M.S. and Ph.D. degrees in electrical engineering from Stanford University, Stanford, CA, in 1998 and 2002, respectively.

Since 2002, he has been an Assistant Professor with the Electrical and Computer Engineering Department, University of Toronto, Toronto, ON, Canada, where he also holds a Canada Research Chair. His main research interests are multiuser information theory, optimization, wireless communications, and broadband access networks.

Prof. Yu is an Editor of the IEEE TRANSACTIONS ON WIRELESS COMMUNICATIONS and a Guest Editor of the EURASIP Journal on Applied Signal Processing (Special Issue on Advanced Signal Processing for Digital Subscriber Lines). 